\title{
PAIR INSTABILITY SUPERNOVAE OF VERY MASSIVE POPULATION III STARS
}

\author{
Ke-Jung Chen $^{1,2,7}$, Alexander Heger ${ }^{3}$, Stan Woosley ${ }^{1}$, Ann Almgren ${ }^{4}$, and Daniel J. Whalen ${ }^{5,6}$ \\ ${ }^{1}$ Department of Astronomy \& Astrophysics, University of California, Santa Cruz, CA 95064, USA; kchen@ ucolick.org \\ ${ }^{2}$ School of Physics and Astronomy, University of Minnesota, Minneapolis, MN 55455, USA \\ ${ }^{3}$ Monash Centre for Astrophysics, Monash University, Victoria 3800, Australia \\ ${ }^{4}$ Center for Computational Sciences and Engineering, Lawrence Berkeley National Lab, Berkeley, CA 94720, USA \\ ${ }^{5}$ T-2, Los Alamos National Laboratory, Los Alamos, NM 87545, USA \\ ${ }^{6}$ Zentrum für Astronomie, Institut für Theoretische Astrophysik, Universität Heidelberg, Albert-Ueberle-Str. 2, D-69120 Heidelberg, Germany \\ Received 2014 February 22; accepted 2014 July 3; published 2014 August 13
}

\begin{abstract}
Numerical studies of primordial star formation suggest that the first stars in the universe may have been very massive. Stellar models indicate that non-rotating Population III stars with initial masses of 140-260 $M_{\odot}$ die as highly energetic pair-instability supernovae. We present new two-dimensional simulations of primordial pair-instability supernovae done with the CASTRO code. Our simulations begin at earlier times than previous multidimensional models, at the onset of core contraction, to capture any dynamical instabilities that may be seeded by core contraction and explosive burning. Such instabilities could enhance explosive yields by mixing hot ash with fuel, thereby accelerating nuclear burning, and affect the spectra of the supernova by dredging up heavy elements from greater depths in the star at early times. Our grid of models includes both blue supergiants and red supergiants over the range in progenitor mass expected for these events. We find that fluid instabilities driven by oxygen and helium burning arise at the upper and lower boundaries of the oxygen shell $\sim 20-100 \mathrm{~s}$ after core bounce. Instabilities driven by burning freeze out after the SN shock exits the helium core. As the shock later propagates through the hydrogen envelope, a strong reverse shock forms that drives the growth of Rayleigh-Taylor instabilities. In red supergiant progenitors, the amplitudes of these instabilities are sufficient to mix the supernova ejecta.
\end{abstract}

Key words: cosmology: theory - early universe - galaxies: formation - galaxies: high-redshift - hydrodynamics quasars: supermassive black holes - stars: early-type - supernovae: general

Online-only material: color figures

\section{INTRODUCTION}

Modern cosmological simulations suggest that the first stars formed in small pre-galactic structures known as cosmological halos with masses of $\sim 10^{6} M_{\odot}$ at $z \sim 20$ (Bromm et al. 1999; Abel et al. 2000, 2002; Bromm et al. 2002; Nakamura \& Umemura 2001; O'Shea \& Norman 2007; Yoshida et al. 2008; Karlsson et al. 2013). The original models suggested that Population III (Pop III) stars were 100-500 $M_{\odot}$ and formed in isolation, one star per halo. Simulations have since shown that some Pop III stars formed in binaries (Turk et al. 2009) or small multiples (Stacy et al. 2010, 2012; Greif et al. 2011, 2012). These and other calculations (Hosokawa et al. 2011; Hirano et al. 2014) indicate that Pop III stars were likely $40-500 M_{\odot}$. The properties of primordial stars are key to understanding early cosmological reionization (Whalen et al. 2004; Kitayama et al. 2004; Abel et al. 2007) and chemical enrichment (Mackey et al. 2003; Santoro \& Shull 2006; Schneider et al. 2006; Smith \& Sigurdsson 2007; Smith et al. 2009; Ritter et al. 2012; SafranekShrader et al. 2014; Cooke \& Madau 2014). These stars also populated the first galaxies (Johnson et al. 2008; Greif et al. 2008; Jeon et al. 2012; Pawlik et al. 2011; Wise et al. 2012; Pawlik et al. 2013; Jeon et al. 2014) and may be the origin of supermassive black holes (SMBHs; Milosavljević et al. 2009; Alvarez et al. 2009; Park \& Ricotti 2011; Johnson et al. 2012; Whalen \& Fryer 2012; Agarwal et al. 2012; Johnson et al. 2013a, 2013b; Latif et al. 2013a, 2013b; Whalen et al. 2013g, 2013h).

The final fate of the first stars depends on their masses. In particular, non-rotating 140-260 $M_{\odot}$ Pop III stars are thought

\footnotetext{
7 IAU Gruber Fellow.
}

to die in highly energetic pair-instability supernovae (PSNe; Barkat et al. 1967; Glatzel et al. 1985; Heger \& Woosley 2002, 2010; rotation can extend this lower limit down to $85 M_{\odot}$; Chatzopoulos \& Wheeler 2012a). Stars in this mass range reach central temperatures above $10^{9} \mathrm{~K}$ at densities below $10^{6} \mathrm{~g} \mathrm{~cm}^{-3}$, which favors the creation of electron-positron pairs at the expense of thermal pressure support in the core. The adiabatic index, $\gamma_{\text {ad }}$, in the core falls below the critical value of four-thirds, causing it to contract. Temperatures and densities in the core rise steeply, triggering explosive burning of oxygen and silicon. The energy release (up to $10^{53} \mathrm{erg}$ ) reverses the core contraction and, in most cases, completely unbinds the star, leaving no compact remnant behind (but see Whalen et al. 2013c). Such events also produce up to $50 M_{\odot}$ of ${ }^{56} \mathrm{Ni}$.

Recent events have rekindled interest in PSNe. PSN are promising candidates for luminous $\mathrm{SNe}$ recently discovered in the local universe, SN 2007bi at $z=0.123$ (Gal-Yam et al. 2009) and SN 2213-1745 at $z=2.05$ (Cooke et al. 2012). However, these events can be also explained by other models such as magnetar spin-down (Kasen \& Bildsten 2010; Woosley 2010; Dessart et al. 2012) or H-poor SN ejecta circumstellar interaction (Chatzopoulos \& Wheeler 2012b; Moriya et al. 2013). The nature of these transits is still under debate.

Stars with masses above the canonical limit of $150 M_{\odot}$ have also been found, including some with masses greater than $300 M_{\odot}$ (Humphreys \& Davidson 1979; Davidson \& Humphreys 1997; Crowther et al. 2010). New studies have also shown that PSNe will be visible in the near infrared (NIR) at $z \sim 15-20$ to the James Webb Space Telescope (JWST), the Wide-Field Infrared Survey Telescope (WFIRST) and the next generation of extremely large telescopes (Kasen et al. 2011; 


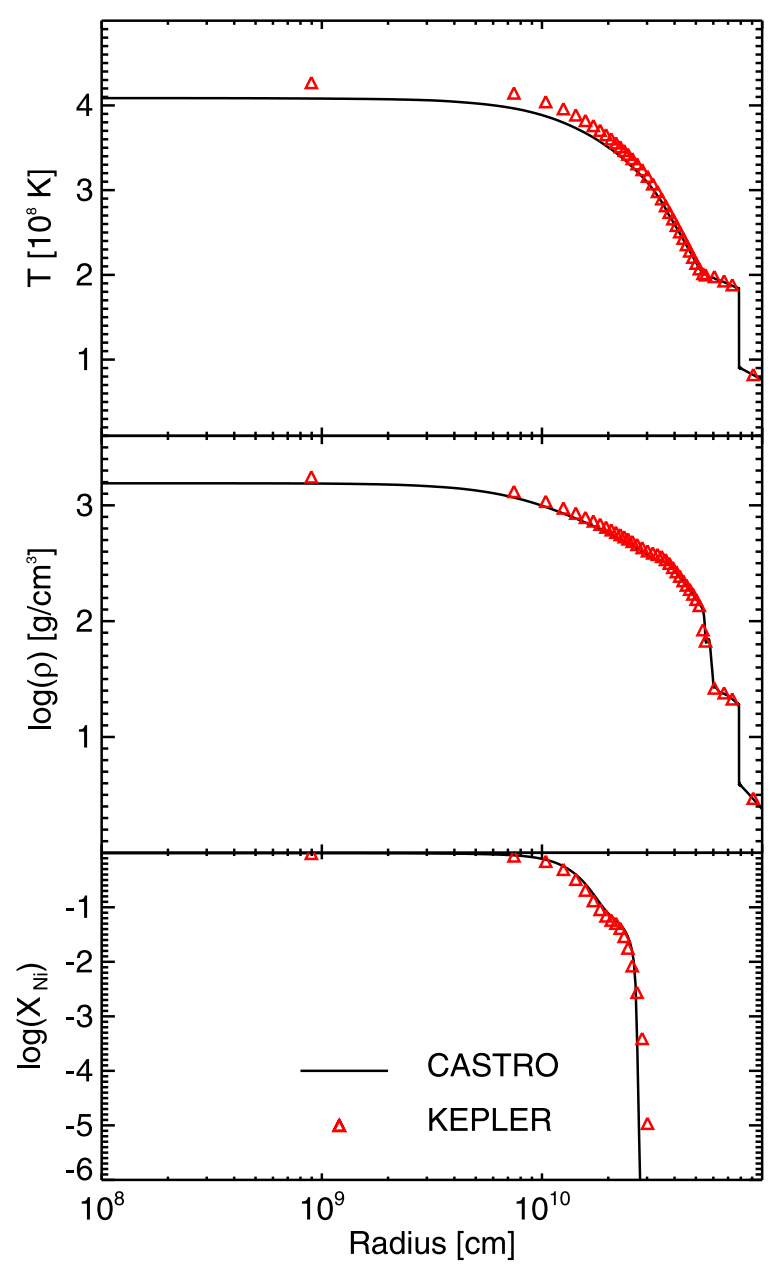

Figure 1. Comparison of explosive burning in the B200 PSN in 1D in KEPLER and CASTRO. The density, temperature, and ${ }^{56} \mathrm{Ni}$ mass fraction profiles are shown at $\sim 100 \mathrm{~s}$. The spatial resolution in CASTRO at the finest level is $10^{8} \mathrm{~cm}$.

(A color version of this figure is available in the online journal.)

Whalen et al. 2013e; Hummel et al. 2012; Pan et al. 2012; Whalen et al. 2013a, 2013i, 2014; de Souza et al. 2013, 2014). PSNe could therefore probe the masses of the first generation of stars (see also Meiksin \& Whalen 2013; Whalen et al. 2013f, 2013b, 2013d; Chatzopoulos \& Wheeler 2012b; Mesler et al. 2014; Chen et al. 2014).

Most PSN models to date have been one-dimensional (1D; Ober et al. 1983; Stringfellow \& Woosley 1988; Scannapieco et al. 2005; Dessart et al. 2013). In the initial stages of a supernova, however, spherical symmetry can be broken by fluid instabilities that require multidimensional simulations. Recent two-dimensional (2D) models of Pop III PSNe by Joggerst \& Whalen (2011) found that in most cases either no instabilities or only mild ones arose in the explosion. However, their models proceeded from KEPLER profiles in which explosive burning was already complete. In reality, instabilities could be seeded at earlier times by core contraction and nuclear burning. They could alter the energetics and chemical yields of the explosion itself by mixing hot ash with fuel and enhancing burning.

Chen et al. (2011) examined initial core contraction and bounce in PSNe in 2D but did not evolve the shock to breakout. Chatzopoulos et al. (2013) modeled the PSNe of rotating stars with different masses in 2D. However, due to the rotation and metallicity effects, their study was limited to PSNe of blue supergiants. Here, we consider the PSNe of non-rotating blue

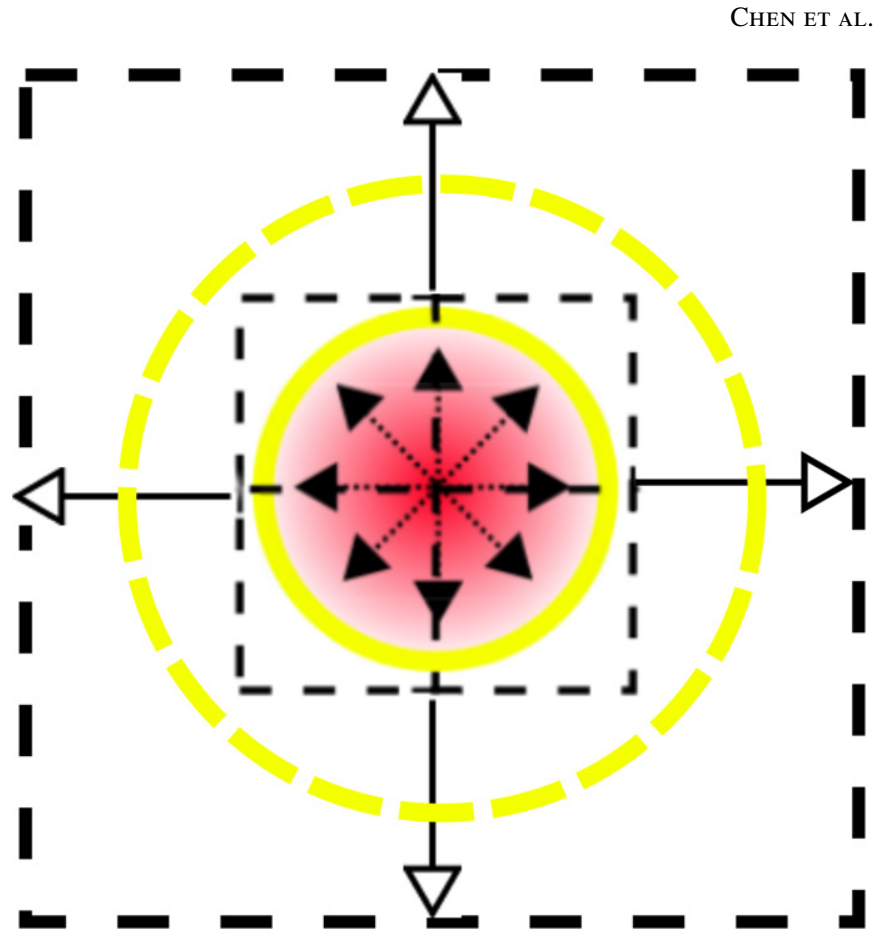

Figure 2. Homographic expansion. The yellow circle denotes the SN shock and the red region is the ejecta. When the simulation is launched just the core of the star resides on the grid in order to resolve explosive burning and the onset of fluid instabilities. When the shock reaches the boundary of the grid (inner rectangle) we expand the grid, mapping the final state of the previous calculation onto the new mesh and grafting onto it the original, undisturbed profile of the star. The shock (dashed yellow circle) is then evolved to the boundary of the new mesh (outer rectangle), and the procedure is repeated.

(A color version of this figure is available in the online journal.)

supergiants and red supergiants from $150-250 M_{\odot}$ in 2D to investigate the formation of dynamical instabilities during the explosion and their impact on energetics and elemental yields. We describe our numerical methods and progenitor models in Section 2. The explosions, dynamical instabilities and internal mixing are examined in Section 3. We conclude in Section 4.

\section{NUMERICAL METHOD}

Self-consistent multidimensional stellar evolution models from the onset of hydrogen burning to eventual core contraction and explosion remain beyond the realm of contemporary computational power. We instead evolve PSN progenitors in the 1D Lagrangian code KEPLER (Weaver et al. 1978; Heger et al. 2001) up to the onset of explosive oxygen burning, just a few tens of seconds before maximum core compression. At this time, the central temperature of stars is about $3.3 \times 10^{9} \mathrm{~K}$ and the explosive silicon burning, which produces most of ${ }^{56} \mathrm{Ni}$ and explosion energy, is about to occur.

We then port the KEPLER profile onto a 2D cylindrical coordinate grid in CASTRO (Almgren et al. 2010; Zhang et al. 2011) and evolve the star through core bounce, explosive burning, and shock breakout from the surface. This approach captures the most important features of the explosion and is computationally tractable.

\subsection{D KEPLER Progenitor Models}

In massive primordial stars, hydrogen burning proceeds by the carbon-nitrogen-oxygen (CNO) cycle as in their metalrich counterparts, except that carbon must first be formed by helium fusion via the triple alpha process. Only very small metal mass fractions are required to initiate the cycle. Typical 


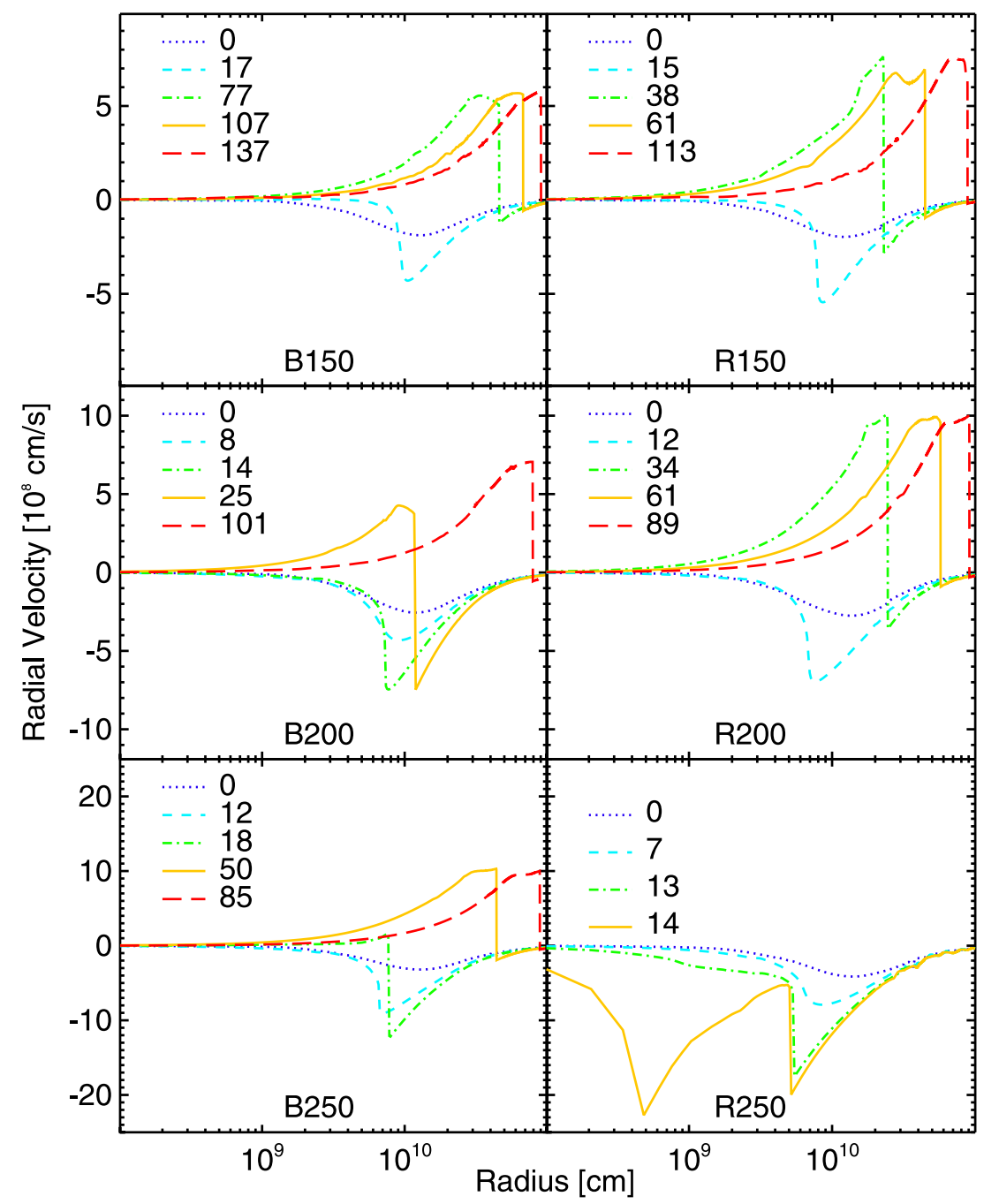

Figure 3. Radial velocity profiles during core bounce. The number for each curve is the time in seconds since the start of the simulation. Except in the R250 run, the reversal of collapse by explosive burning is evident.

(A color version of this figure is available in the online journal.)

$\mathrm{CNO}$ mass fractions are $10^{-9}$ for central $\mathrm{H}$ burning and $10^{-7}$ for $\mathrm{H}$ shell burning. The $\mathrm{CNO}$ cycle proceeds at a higher density and temperature, and overall lower entropy, than at higher metallicities. Unlike metal-rich stars, primordial stars have a very small entropy barrier between the hydrogen shell and the helium core during helium burning. In massive stars, radiation dominates the pressure and facilitates convection. The central convection zone, which is rich in carbon and oxygen, can come close to the hydrogen-burning shell and even mix with it if there is sufficient convective overshoot or other convective boundary mixing (Meakin \& Arnett 2007; Arnett et al. 2009; Woodward et al. 2013).

If mixing occurs, then carbon causes hydrogen to burn at a much higher rate, which is proportional to the enrichment in $\mathrm{CNO}$, if temperatures and densities were fixed. Depending on the degree of mixing, the higher burning rates can inflate the $\mathrm{H}$ envelope by about an order of magnitude in radius and turn the star into a red supergiant. If convective overshoot is weak, the star does not expand, and it evolves into a blue supergiant instead. The degree of mixing in any given star cannot be predicted a priori and is usually a free parameter in 1D models (Marigo et al. 2001; Heger et al. 2002; Scannapieco et al. 2005; Woosley et al. 2010).
We adopt 150,200 , and $250 M_{\odot}$ non-rotating progenitor models from Heger \& Woosley (2002); Scannapieco et al. (2005); Heger \& Woosley (2010) for our simulation suite. They were evolved with either weak or strong convective mixing and therefore die as blue supergiants or red supergiants whose internal structures bracket those expected for very massive primordial stars. Our models are designated as XYYY, where $\mathrm{X}$ indicates if the star is a red (R) or blue (B) supergiant and YYY is the mass of the star $\left(150,200\right.$, or $\left.250 M_{\odot}\right)$. As with the usual convention that massive Pop III stars do not lose mass over their lives (Kudritzki 2000; Vink et al. 2001; Baraffe et al. 2001; Krtička \& Kubát 2006; Ekström et al. 2008), mass loss is turned off in our models. We summarize the properties of the stars in Table 1.

\subsection{CASTRO}

CASTRO is a massively parallel, multidimensional Eulerian adaptive mesh refinement (AMR) radiation hydrodynamics code for astrophysical applications. CASTRO has an unsplit piecewiseparabolic method (PPM) hydro scheme (Steiner \& Gautschy 1998) and block-structured AMR. We use the Helmholtz equation of state (Timmes \& Swesty 2000), which includes 


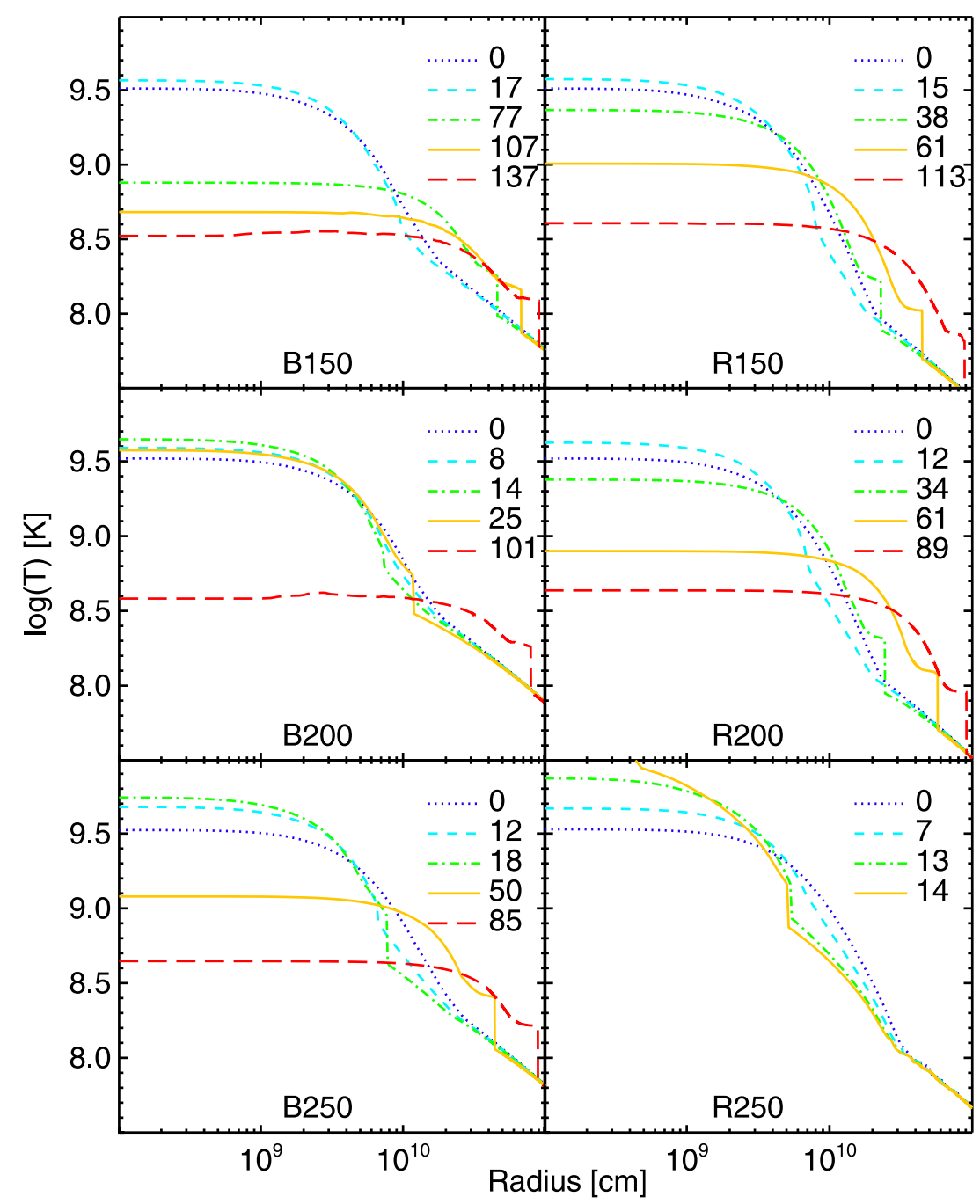

Figure 4. Radial temperature profiles during core bounce. The number for each curve is the time in seconds in the CASTRO run. When the shock reaches $10^{11} \mathrm{~cm}$, post-shock temperatures have dropped to $3 \sim 10^{8} \mathrm{~K}$, except in the R250 run in which they continue to rise, suggesting complete collapse to a black hole.

(A color version of this figure is available in the online journal.)

Table 1

Progenitor Models

\begin{tabular}{lccccc}
\hline \hline Name & $\begin{array}{c}M_{*} \\
\left(M_{\odot}\right)\end{array}$ & $\begin{array}{c}M_{\mathrm{He}} \\
\left(M_{\odot}\right)\end{array}$ & $\begin{array}{c}\rho_{\mathrm{c}} \\
\left(10^{6} \mathrm{~g} \mathrm{~cm}^{-3}\right)\end{array}$ & $\begin{array}{c}T_{\mathrm{c}} \\
\left(10^{9} \mathrm{~K}\right)\end{array}$ & $\begin{array}{c}R \\
\left(10^{13} \mathrm{~cm}\right)\end{array}$ \\
\hline B150 & 150 & 67 & 1.40 & 3.25 & 16.54 \\
B200 & 200 & 95 & 1.23 & 3.31 & 2.86 \\
B250 & 250 & 109 & 1.11 & 3.34 & 23.06 \\
R150 & 150 & 59 & 1.58 & 3.25 & 25.69 \\
R200 & 200 & 86 & 1.27 & 3.31 & 27.68 \\
R250 & 250 & 156 & 0.95 & 3.38 & 20.76 \\
\hline
\end{tabular}

Notes. $M_{*}$ : initial stellar mass; $M_{\mathrm{He}}$ : helium core mass; $\rho_{\mathrm{c}}$ : central density; $T_{\mathrm{c}}$ : central temperature; $R$ : stellar radius.

contributions by both degenerate and non-degenerate relativistic and non-relativistic electrons, electron-positron pairs, ions and radiation. The monopole approximation is used for self-gravity, in which a spherically symmetric gravitational potential is constructed from the radial average of the density and then applied to gravitational force updates everywhere in the AMR hierarchy (Almgren et al. 2010). Even with dynamical instabilities, this is a reasonable approximation to the matter distribution of the star and is very efficient. Our models include multispecies advection for the 19 elements listed below.

\subsection{Nuclear Reaction Network}

We implemented the 19-isotope APPROX reaction network in CASTRO to follow nuclear burning (Weaver et al. 1978; Timmes 1999). This is the same network used in our KEPLER models, and it evolves mass fractions for ${ }^{1} \mathrm{H},{ }^{3} \mathrm{He},{ }^{4} \mathrm{He},{ }^{12} \mathrm{C},{ }^{14} \mathrm{~N},{ }^{16} \mathrm{O},{ }^{20} \mathrm{Ne}$, ${ }^{24} \mathrm{Mg},{ }^{28} \mathrm{Si},{ }^{32} \mathrm{~S},{ }^{36} \mathrm{Ar},{ }^{40} \mathrm{Ca},{ }^{44} \mathrm{Ti},{ }^{48} \mathrm{Cr},{ }^{52} \mathrm{Fe},{ }^{54} \mathrm{Fe},{ }^{56} \mathrm{Ni}$, protons (due to photo-disintegrations), and neutrons. It includes alphachain reactions, a heavy-ion reaction network, hydrogen burning cycles, photo-disintegration of heavy nuclei, and energy loss due to thermal neutrinos. Nuclear burning is self-consistently evolved with hydrodynamics. Since explosive burning of ${ }^{4} \mathrm{He}$, ${ }^{16} \mathrm{O}$, and ${ }^{28} \mathrm{Si}$ is what primarily drives the energetics and yields in our PSN models, this network is sufficient for capturing the energy of the explosion and the synthesis of key isotopes. The most powerful of our explosions produces up to $30 M_{\odot}$ of ${ }^{56} \mathrm{Ni}$. Our network also includes energy release from radioactive decay of ${ }^{56} \mathrm{Ni} \rightarrow{ }^{56} \mathrm{Co} \rightarrow{ }^{56} \mathrm{Fe}$.

To test the APPROX network in CASTRO, we ran the B200 PSN in KEPLER and CASTRO in 1D for about $100 \mathrm{~s}$. As shown in Figure 1, temperatures, densities, and ${ }^{56} \mathrm{Ni}$ yields for the two 


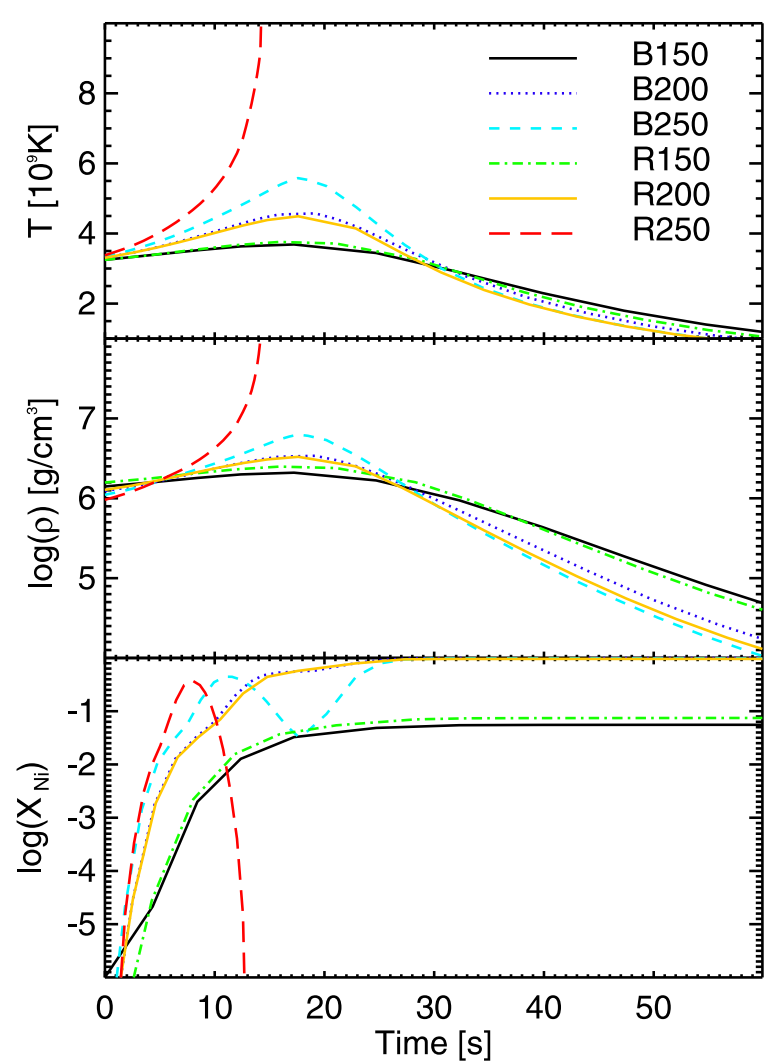

Figure 5. Evolution of $\rho_{c}, T_{c}$, and central ${ }^{56} \mathrm{Ni}$ mass fraction in the first minute of each run. In the first $20 \mathrm{~s},{ }^{56} \mathrm{Ni}$ builds up rapidly in the core from ${ }^{28} \mathrm{Si}$ burning. From 10-20 s, the ${ }^{56} \mathrm{Ni}$ in R250 disappears because it is photodisintegrated. In $\mathrm{B} 250$, the explosive silicon burning creates the first peak, then similar to $\mathrm{R} 250$, some ${ }^{56} \mathrm{Ni}$ is photo-disintegrated. After the core bounces and the central temperature drops. Light elements can recombine to form ${ }^{56} \mathrm{Ni}$ through $\alpha$ capture reactions and result in the second peak.

(A color version of this figure is available in the online journal.)

runs are in good agreement, with a deviation in final explosion energy of less than $1 \%$.

\subsection{Mapping and Initial Setup}

Differences between codes in dimensionality and coordinate mesh can lead to numerical artifacts such as violation of conservation of mass, energy, and momentum when mapping a blast profile from one code to another. The simplest approach is to initialize fluid variables at a given point on a $2 \mathrm{D}$ grid by linearly interpolating between those on the 1D grid that bracket it in radius. However, this practice can fail to resolve critical length scales in the original stellar model, such as those associated with nuclear burning. This is especially true when porting profiles from 1D Lagrangian codes, which can resolve very small features with a just a few zones in mass, to Eulerian grids that may require far more mesh points to resolve the same features in space. Even minor violations in conservation can lead to serious errors in simulations because some processes, like nuclear burning, are very sensitive to temperature. Slight errors in the mapping process can therefore lead to very different outcomes in a run.

We therefore use a new procedure to map our 1D PSN profiles from KEPLER onto 2D grids in CASTRO that preserves the conservation of fluid variables at any resolution (Chen et al. 2012). The grid is then seeded with turbulent perturbations from a Kolmogorov spectrum (Chen et al. 2013) rather than the simple random perturbations used in earlier work to approximate the convective velocities that would be present in a star. Our CASTRO root grid is a $2 \mathrm{D}$ cylindrical coordinate mesh with 256 zones in both $r$ and $z$. Up to three levels of AMR refinement are allowed, each of which is a factor of four greater in resolution, for a maximum resolution 64 times that of the coarse grid. The grid is refined on gradients in density, velocity, and pressure. Because we simulate only one quadrant domain of the star, reflecting and outflow boundary conditions are used on the lower and upper boundaries of the grid, respectively, in both $r$ and $z$.

\subsection{Effective Resolution}

Simulations with nuclear burning are very different from purely hydrodynamical ones because much higher spatial and temporal resolution is required to resolve the scales on which burning occurs. Since nuclear burning is very sensitive to temperature, errors in energy release and nucleosynthesis rates can easily arise in regions that are not fully resolved. We performed a series of runs in 1D in CASTRO to determine the resolution required to resolve burning in our 2D models. In each 1D run, we evolved the star from initial core contraction until the end of all explosive burning, and then computed the total energy of the SN by summing the gravitational, internal, and kinetic energies. The total energy converged at a grid resolution of $10^{9} \mathrm{~cm}$, but because the required resolution may vary slightly in different models we adopted a more conservative resolution of $10^{8} \mathrm{~cm}$ for our 2D CASTRO runs.

In principle, it would be possible to accommodate the entire star on the grid, whose radius can be up to a few times $10^{14} \mathrm{~cm}$, and still resolve explosive burning. However, six levels of AMR refinement would be required to achieve the required dynamical range of $10^{6}$ instead of three, and the manner in which time steps between adjacent levels are sub-cycled in CASTRO causes the simulation to run much more slowly if more than 5 levels are used. As a result, even a 2D simulation of the entire star would require 500,000 CPU hours. Instead of modeling the entire star at once, the initial coarse grid encloses just the core of the star with enough zones to resolve explosive burning. When the SN shock reaches the boundary of the grid we halt the simulation, resample the blast profile onto a larger mesh, and then restart the run. We map the original profile of the star from the radius of the shock to the new outer boundary onto the grid prior to relaunching the run. We repeat this procedure until the entire star resides on the grid. In each regrid, we retain the same total number of grids, as illustrated in Figure 2. We want to simulate a domain about 10 times larger than the size of star which requires eight levels AMR. By using the homographic expansion, we can break one simulation of eight levels AMR into five simulations of three levels AMR. This increases the stability of simulations and saves $\sim\left(2^{5}-5\right) / 2^{5} \sim 84.3 \%$ of CPU time.

The travel time of the shock through the star is at most a few days, much less than the timescales on which the star itself evolves (a few thousand years). It is therefore reasonable to assume that the envelope of the star does not change in the time it takes for the shock to break out of the star. This can also be seen from the free-fall time of the envelope, $T_{\mathrm{ff}} \approx \sqrt{1 / G \rho}$, where $G$ is the gravitational constant and $\rho$ is the density. If we take the density of the envelope to be $10^{-12} \mathrm{~g} \mathrm{~cm}^{-3}$, we find that $T_{\mathrm{ff}}$ is a few hundred years, which is much longer than the time it takes the shock to cross the star. Furthermore, although the spatial resolution decreases after each expansion of the grid, it does not affect the simulation at later times because burning is finished before the first expansion and the dynamical instabilities 

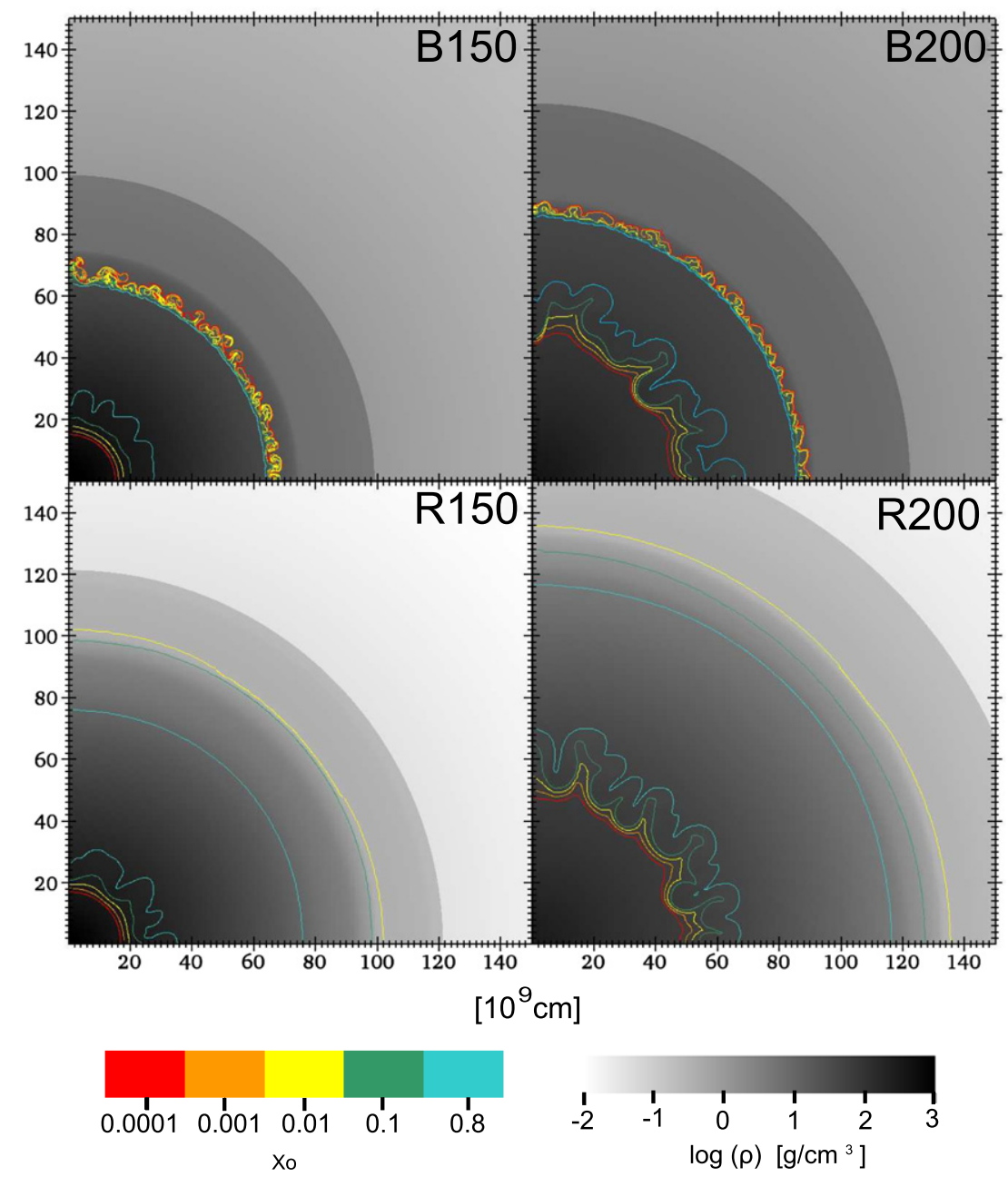

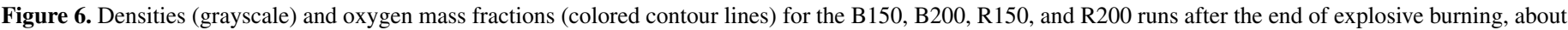

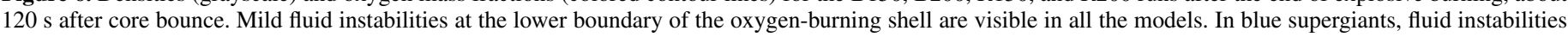
(mixed contours) also appear at the interface between the oxygen/carbon and helium layers.

(A color version of this figure is available in the online journal.)

are well resolved by later grids. Minor sound waves can appear at the boundaries as numerical artifacts of the regrid process. But the shock has a much higher Mach number, $\mathcal{M} \gtrsim 10$, and reaches the outer boundaries before the acoustic waves can affect either explosive burning or the formation of instabilities. Periodically enlarging the grid throughout the run with fewer levels of refinement allows us to evolve the PSN in much shorter simulation times while maintaining full fidelity to the solution.

\section{EXPLOSION}

At the onset of core contraction in CASTRO, energy loss by emission of neutrinos from pair production exceeds energy production by nuclear burning. A few seconds later, the core reaches a temperature $T \sim 4 \times 10^{9} \mathrm{~K}$, which ignites silicon burning. Central silicon burning and oxygen and carbon shell burning proceed out of hydrodynamical equilibrium. Explosive burning lasts 10-20 s but releases enough energy to reverse the ram pressure of core contraction and drive a shock that completely disperses the star. In Figure 3 we show spherically averaged $1 \mathrm{D}$ velocities from the beginning of core contraction to bounce. The outer layers of the core begin to contraction at $2-5 \times 10^{8} \mathrm{~cm} \mathrm{~s}^{-1}$ and then accelerate in a free fall to several $10^{9} \mathrm{~cm} \mathrm{~s}^{-1}$ prior to reversal by explosive burning. In the R250 run, infall simply continues, accelerating to $\sim 2 \times 10^{9} \mathrm{~cm} \mathrm{~s}^{-1}$, or nearly $10 \%$ of the speed of light. In this model, most of the energy from burning goes to photo-disintegration of heavy nuclei rather than a shock, and the star likely collapses to a black hole. In KEPLER simulations, this star develops a large helium core, $\sim 156 M_{\odot}$ and eventually dies as a black hole (Heger \& Woosley 2002). The final mass of black hole is close to $250 M_{\odot}$, because it accretes the entire star.

We show the temperature evolution of the core from contraction to core bounce in Figure 4. The temperature rises during the contraction of the core and then quickly falls after explosive burning. The peak core temperature at bounce is $3-4 \times 10^{9} \mathrm{~K}$, igniting burning of silicon to ${ }^{56} \mathrm{Ni}$. Most of the ${ }^{56} \mathrm{Ni}$ is synthesized at the center of the core, where temperatures and densities are greatest. The evolution of the central temperature, $T_{c}$, density, $\rho_{c}$, and ${ }^{56} \mathrm{Ni}$ mass fraction in the first minute is shown in Figure 5. Both $T_{c}$ and $\rho_{c}$ rise during core contraction. At $15-20 \mathrm{~s}$, $\rho_{c}$ and $T_{c}$ reach their peak values and then fall as the explosion disrupts the core. The higher $T_{c}$ and $\rho_{c}$ in more massive models favor the production of ${ }^{56} \mathrm{Ni}$. In the R250 run, the creation and subsequent destruction of ${ }^{56} \mathrm{Ni}$ together with the runaway $\rho_{c}$ and $T_{c}$ are due to the photo-disintegration of the core and likely creation of a black hole. Explosion energies and ${ }^{56} \mathrm{Ni}$ production for all the models are summarized in Table 2. Unlike numeri- 


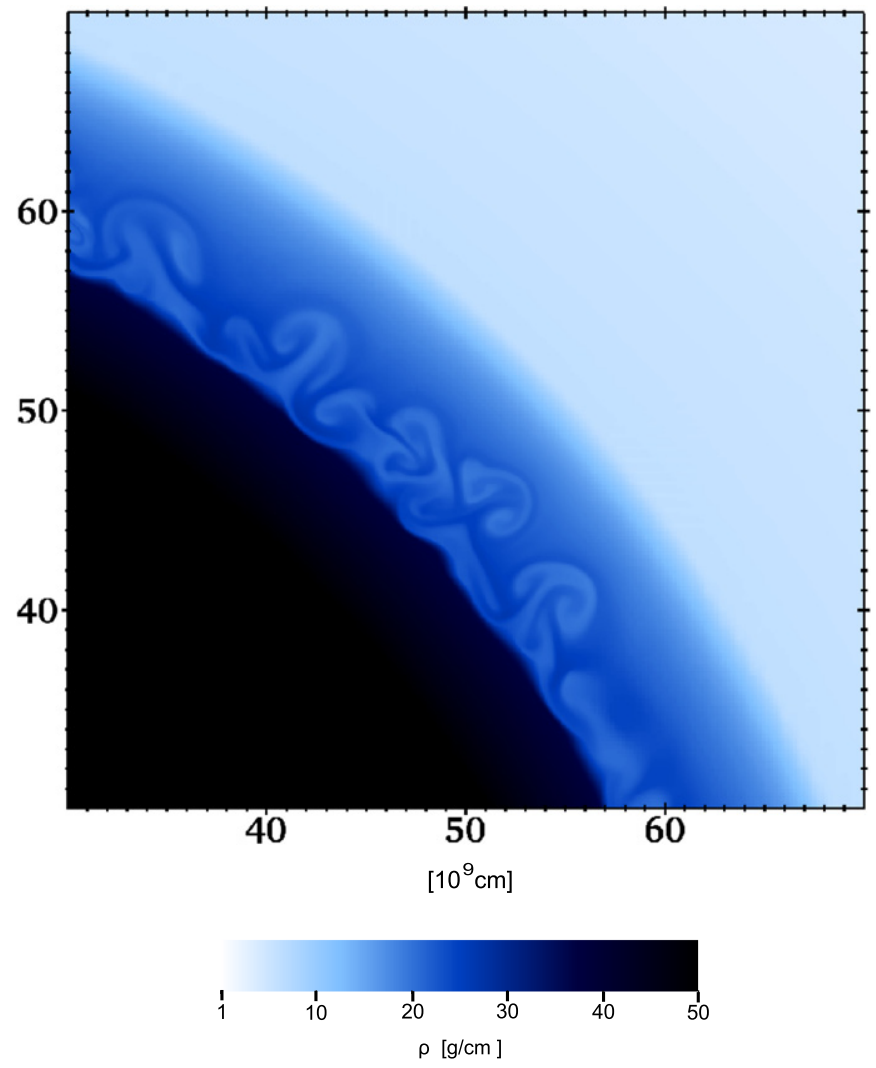

Figure 7. Fluid instabilities due to helium burning. In blue supergiants, the interface between the oxygen and helium shells becomes unstable due to helium burning driven by the shock. Mixing between the two layers continues until the post shock temperature drops below about $3 \times 10^{8} \mathrm{~K}$.

(A color version of this figure is available in the online journal.)

Table 2

${ }^{56} \mathrm{Ni}$ Yields and Explosion Energies

\begin{tabular}{lcr}
\hline \hline Model & $\begin{array}{c}E \\
\left(10^{52} \mathrm{erg}\right)\end{array}$ & $\begin{array}{c}M_{\mathrm{Ni}} \\
\left(M_{\odot}\right)\end{array}$ \\
\hline B150 & 1.29 & 0.07 \\
B200 & 4.14 & 6.57 \\
B250 & 7.23 & 28.05 \\
R150 & 1.19 & 0.10 \\
R200 & 3.43 & 4.66 \\
R250 & $\cdots$ & $\cdots$ \\
\hline
\end{tabular}

cal simulations of core-collapse SNe, the explosion mechanics of thermonuclear PSNe are insensitive to the dimensionality of simulations because of the nature of thermonuclear explosion. In the case of PSN, not much extra burning are generated through mixing. The 1D PSN models that explode in KEPLER (Heger \& Woosley 2002) also explode in CASTRO. The explosion energetics and yields are very similar between 1D and 2D models.

\subsection{Fluid Instabilities Triggered by Burning}

Do fluid instabilities or mixing occur in this short but violent phase of the PSN? We show densities and oxygen abundances for all six models at the end of explosive burning in Figure 6. During the core contraction, fluid instabilities triggered by burning arise at the inner boundary of the oxygen-burning shell and are visible in the inner contours of Figure 6. However, they do not develop amplitudes that are large enough to transport ${ }^{56} \mathrm{Ni}$ to the upper layers of the shell. Burning in the ${ }^{28} \mathrm{Si}$ core does not trigger visible instabilities in any of the models. They

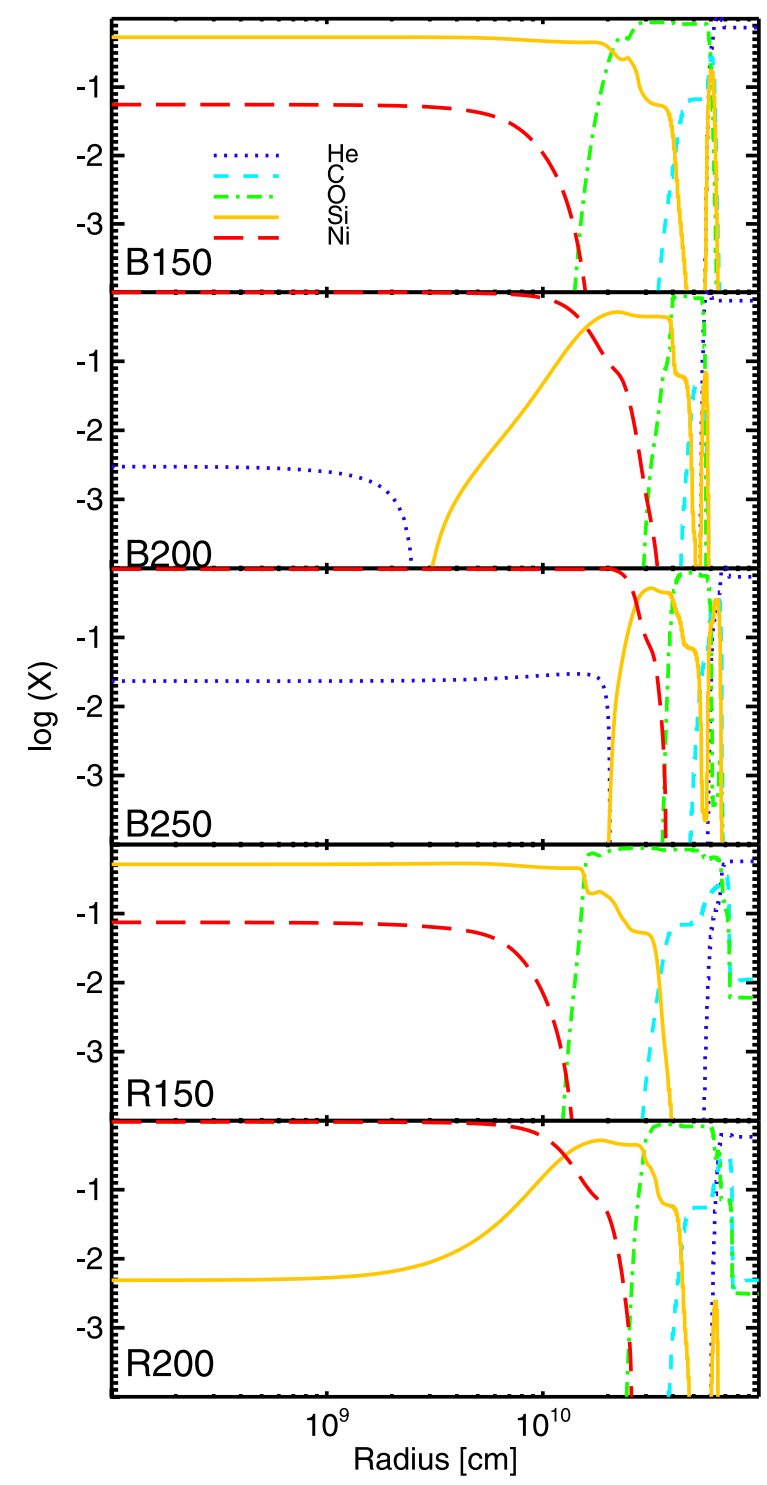

Figure 8. Spherically averaged mass fractions for $\mathrm{H}, \mathrm{C}, \mathrm{O}, \mathrm{Si}$, and $\mathrm{Ni}$ as a function of radius at $100 \mathrm{~s}$, after nuclear burning is complete. More massive stars produce more ${ }^{56} \mathrm{Ni}$ and thinner oxygen burning shells. The B200 and B250 runs show traces of ${ }^{4} \mathrm{He}$ in the inner regions from the photo-disintegration of ${ }^{56} \mathrm{Ni}$ during core bounce.

(A color version of this figure is available in the online journal.)

may not appear because the density and temperature profiles for $r<10^{9} \mathrm{~cm}$, where ${ }^{56} \mathrm{Ni}$ is created, are nearly flat, and this plus the absence of any interface or discontinuities may suppress their formation.

Nuclear burning (of mostly helium) by the shock at the interface between the oxygen/carbon and helium shells enhances the entropy gradient across them and triggers the formation of instabilities there, as seen in the outer contours in the B150 and B200 runs. They freeze out about $100 \mathrm{~s}$ after the shock reaches the helium-rich envelope. We show an enlarged view of these features in Figure 7. These interfaces remain stable in the R150 and R200 runs because the shocks are less energetic and the helium shells are thinner. Explosive burning is largely quenched thereafter because temperatures become too low to sustain it. In Figure 8, spherically averaged mass fractions are shown for selected isotopes. No ${ }^{56} \mathrm{Ni}$ (the red dashed line) has been dredged up to the oxygen-burning shell (the green dot-dashed line). 


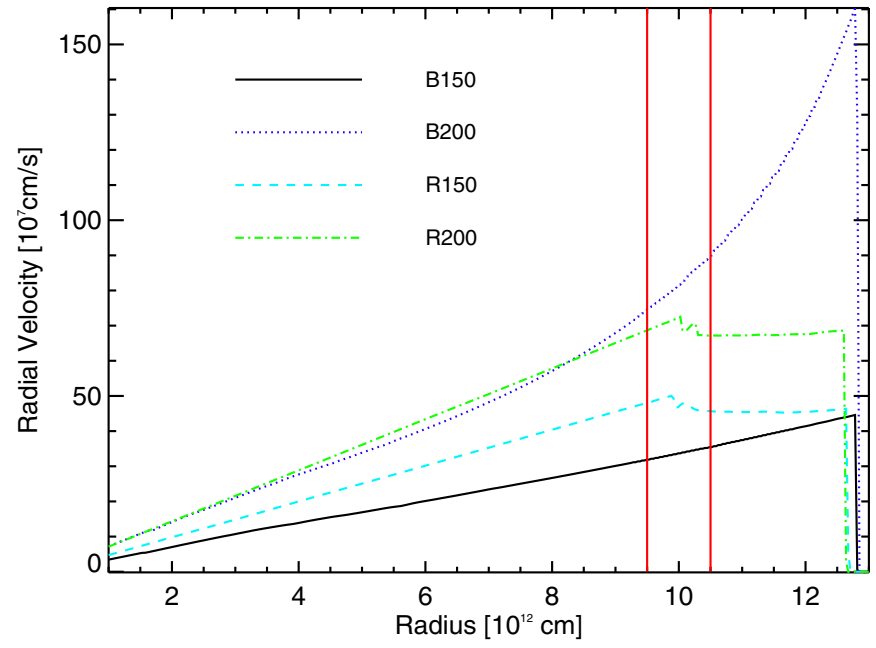

Figure 9. Radial velocity profiles when the shock enters the hydrogen-rich envelope. Reverse shocks can be seen between the two vertical red in the red supergiants.

(A color version of this figure is available in the online journal.)

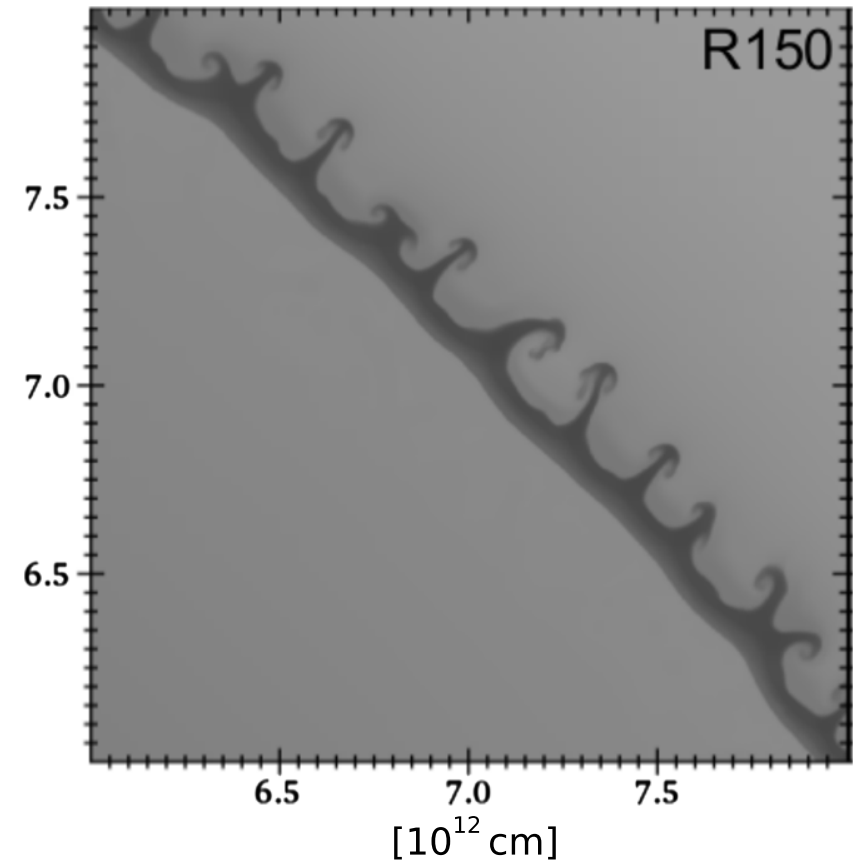

Figure 11. Enlarged view of the RT fingers in Figure 10. These fingers appear right after the formation of reverse shock and they have overdensities of about 10 .
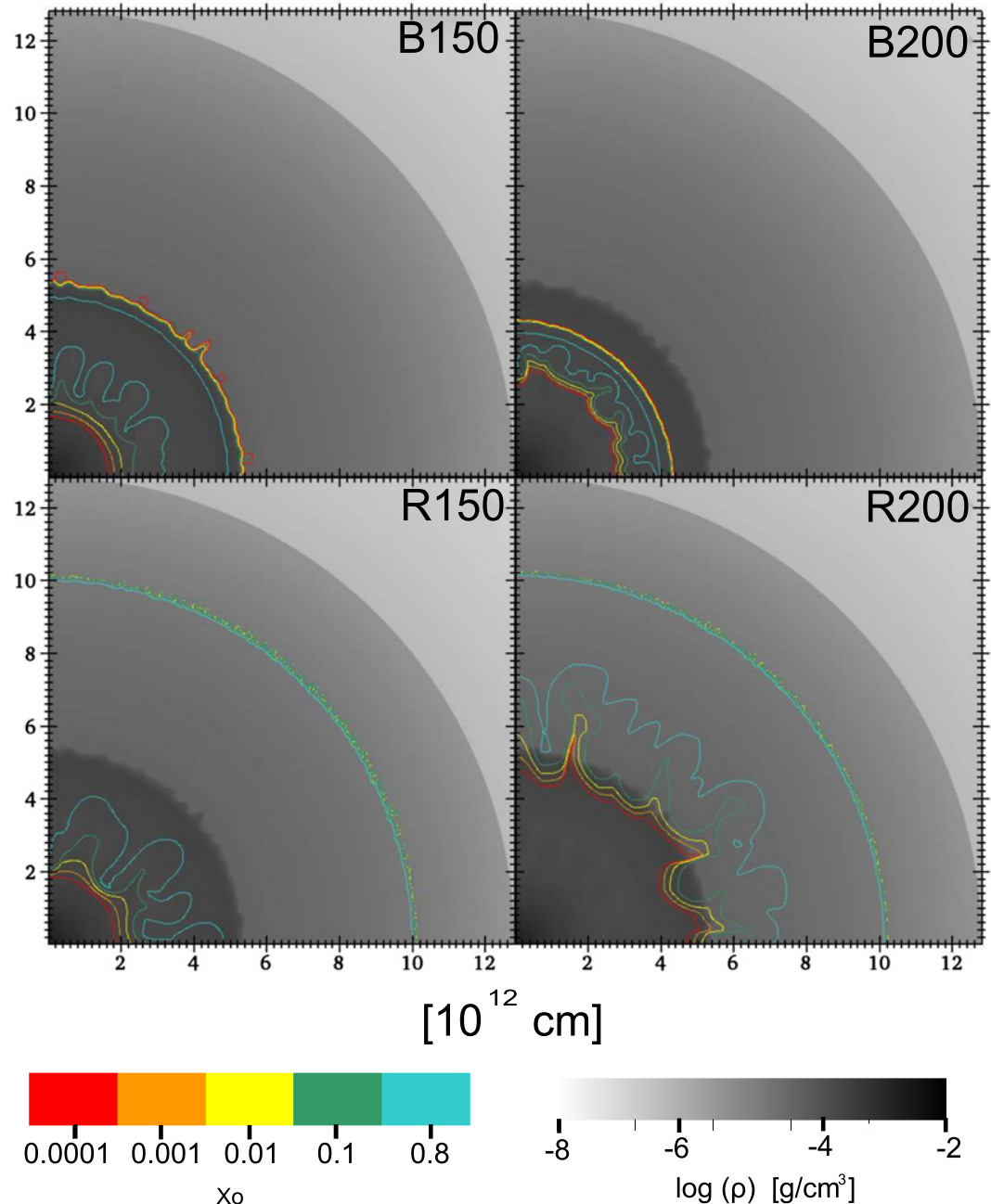

Figure 10. Densities (grayscale) and oxygen mass fractions (color contour lines) when the shock enters the hydrogen envelope. In red supergiants, a reverse shock forms, causing the growth of the RT instabilities, which are visible in the outer green contours. No RT instabilities form in the blue supergiants.

(A color version of this figure is available in the online journal.) 


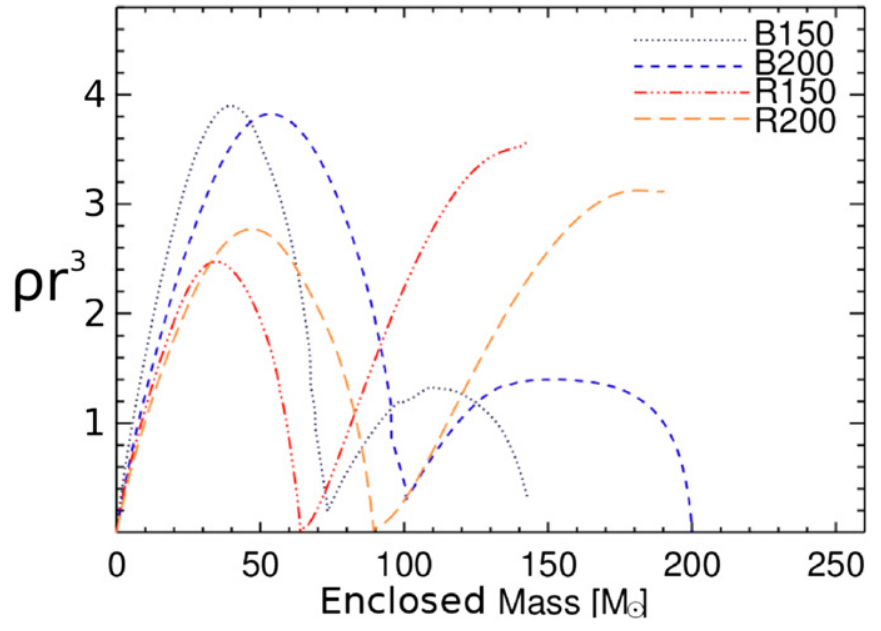

Figure 12. $\rho r^{3}$ for the stellar profiles in mass coordinate. The $y$ axis represents normalized mass. Each model features two peaks, the first is the helium core and the second is the hydrogen envelope. The red supergiants have an extended outer envelope and larger second peak. A reverse shock forms when the forward shock propagates into the region of the second peak. Since the forward shock forms at the edge of helium core, no reverse shock forms in the first peak.

(A color version of this figure is available in the online journal.)

\subsection{Instabilities due to the Reverse Shock}

As the shock begins to plow up the hydrogen envelope, it decelerates, creating a reverse shock. If the gas pressure, $P$, and the density, $\rho$, satisfy the Rayleigh-Taylor (RT) criterion for a fluid (Chandrasekhar 1961)

$$
\frac{d P}{d r} \frac{d \rho}{d r}<0
$$

then instabilities will form. For a strong adiabatic shock in a power-law density profile, $\rho=A r^{w}$, the flow becomes selfsimilar and any of its variables can be expressed as a function, $f_{w}(A, E, t)$, of explosion energy, $E$, and time, $t$ (Sedov 1959; Herant \& Woosley 1994). The shock velocity can then be obtained from dimensional analysis:

$$
V_{\mathrm{s}}=A^{\frac{-1}{(5+w)}} E^{\frac{1}{(w+5)}} t^{\frac{-(w+3)}{5+w}} .
$$

The evolution of the shock velocity depends on $w$. If $w=-3$, the shock velocity is independent of time. If $w<-3$, the hot gas at high pressure behind the shock accelerates the shock. If $w>-3$, the material swept up by the shock decelerates the shock. This deceleration is communicated to the fluid behind by the shock at the sound speed, and it sets up a pressure gradient in the direction that the material was decelerated. The sound waves generated by the deceleration can steepen this pressure gradient and become a reverse shock. The reverse shock grows in spatial coordinate but recedes in mass coordinate. In Figure 9, we show velocity profiles for our models when the shock enters the hydrogen-rich envelope. Reverse shocks are clearly present in red supergiants but not blue supergiants. When the density gradient is in the opposite direction of the pressure gradient, the contact discontinuity between the ejecta and the envelope becomes unstable and RT fingers form. In Figure 10, we show

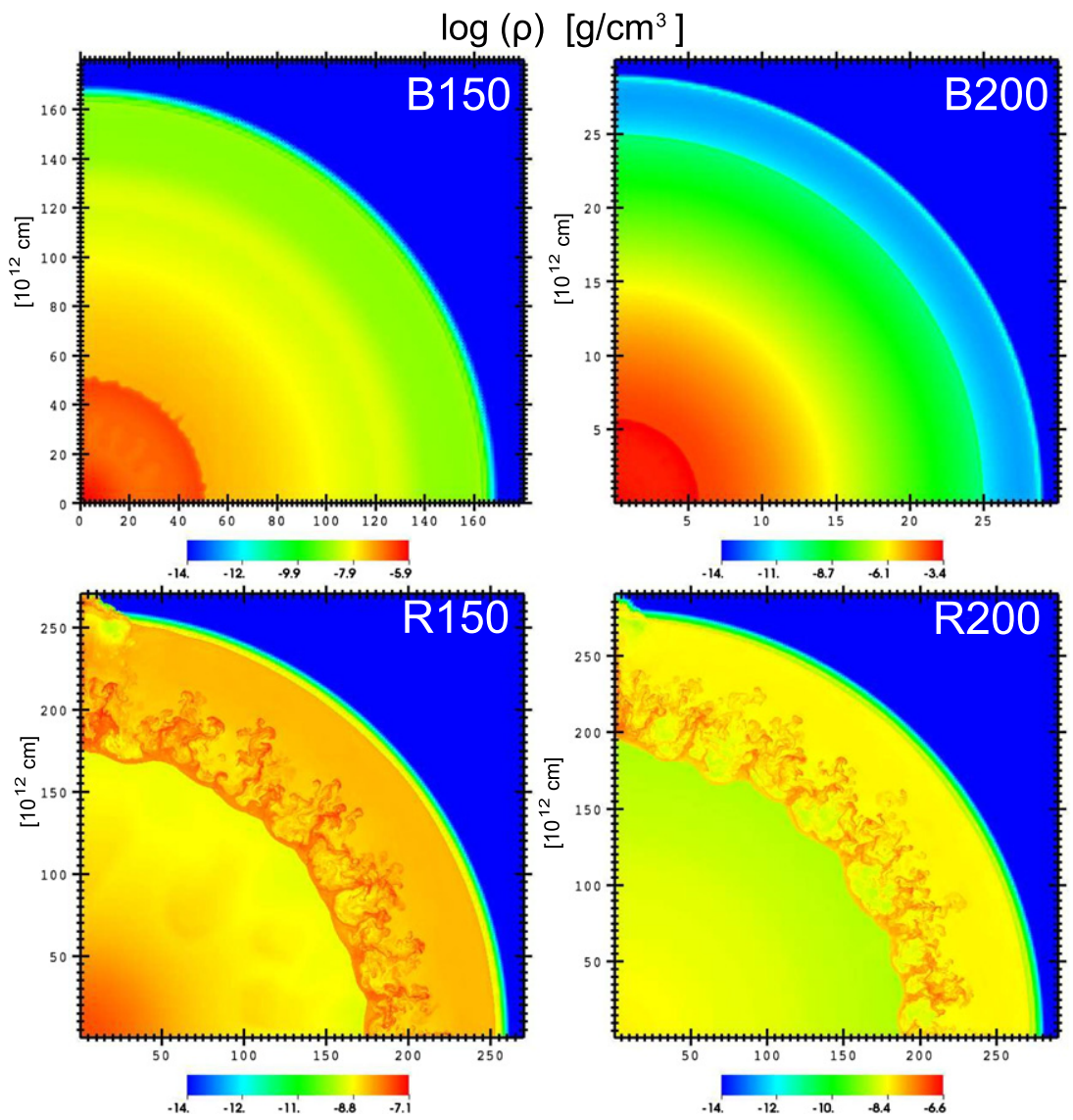

Figure 13. Densities just before shock breakout. In red supergiants, fluid instabilities due to the reverse shock have devolved into turbulence and large-scale mixing. (A color version of this figure is available in the online journal.) 


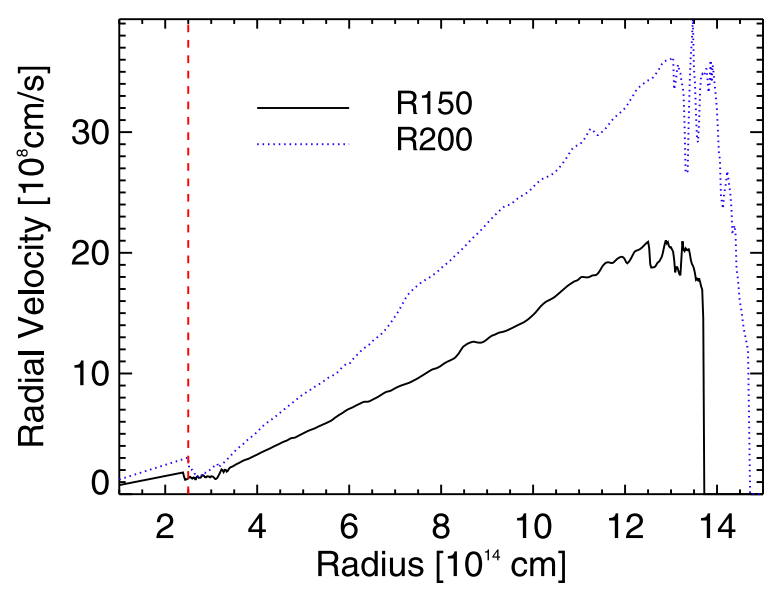

Figure 14. Radial velocity profiles after shock breakout in the red supergiants. The forward shock rapidly accelerates in the low-density CSM. Without the deceleration of the forward shock, the reverse shock loses pressure support and dissipates. Mixing then freezes out. The red-dashed line marks the original radius of the stars, about $2.5 \times 10^{14} \mathrm{~cm}$.

(A color version of this figure is available in the online journal.)

densities and contours for oxygen mass fractions when the shock enters the hydrogen envelope. Dynamical instabilities with small amplitudes are visible in the outer green contours of the red supergiants. The clumpy structures created by the RT instabilities have overdensities of about ten. We show an enlarged view of these features in Figure 11. From Equation (1), a reverse shock only forms in regions of increasing $\rho r^{3}$. We show $\rho r^{3}$ as a function of radius in Figure 12. The two peaks in each star are its helium core and hydrogen envelope, respectively. Because the red supergiants have more extended hydrogen envelopes than the blue supergiants, they have much higher second peaks that favor the formation of reverse shocks and the development of RT instabilities.

The instabilities due to the reverse shock grow until the forward shock breaks out of the star. Their growth is enhanced by Kelvin-Helmholtz instabilities that are induced by shear flows, and both types of instability can efficiently mix ejecta in PSNe of red supergiants. Gas densities just before shock breakout are shown in Figure 13. PSNe of blue supergiants expand almost homologously. However, explosions of red supergiants exhibit significant mixing over a distance of $\sim 5 \times 10^{13} \mathrm{~cm}$, or about $20 \%$ of their radii. The hydrogen, helium, carbon, and oxygen shells all become blended together. We allow the PSN to expand to about eight times the radius of the star in a uniform circumstellar medium (CSM) with a density of $10^{-18} \mathrm{~g} \mathrm{~cm}^{-3}$, $\sim 10,000$ times lower than at the surface of the star. Mixing halts shortly after breakout. Velocity profiles for the ejecta are shown in Figure 14. The forward shock accelerates in the low-density CSM. Without the deceleration of the forward shock, the reverse shock loses pressure support and dissipates, and the instabilities cease to evolve.

Mass-weighted mass fractions for ${ }^{12} \mathrm{C},{ }^{16} \mathrm{O},{ }^{24} \mathrm{Mg},{ }^{28} \mathrm{Si}$, and ${ }^{56} \mathrm{Ni}$ are plotted in Figure 15, which confirms that these elements are mostly segregated in blue supergiants but mixed together in red supergiants. However, even in red supergiants, only a little ${ }^{56} \mathrm{Ni}$ is dredged up from lower layers, so it is unlikely that much $\gamma$ ray emission from ${ }^{56} \mathrm{Ni}$ decay would be detected from PSNe. Most of the energy due to ${ }^{56} \mathrm{Ni}$ decay is instead deposited as thermal energy in the ejecta.

\section{DISCUSSION AND CONCLUSION}

Our simulations of Pop III PSNe are the first to follow core contraction, nuclear burning, explosion and the end of mixing in $2 \mathrm{D}$ in both red and blue supergiants. As mentioned earlier, instabilities seeded during core contraction and explosive burning in principle can alter the yield of the SN by mixing hot ash with fuel and accelerating burning, unlike instabilities that develop in the reverse shock at later times after the end of burning. Although instabilities do appear at these early stages, they do not affect nucleosynthesis or energy generation for two reasons. First, most of the explosive silicon burning occurs at the very center of the star, not at the base of the oxygen-burning shell where the instabilities form, so there is no evidence of

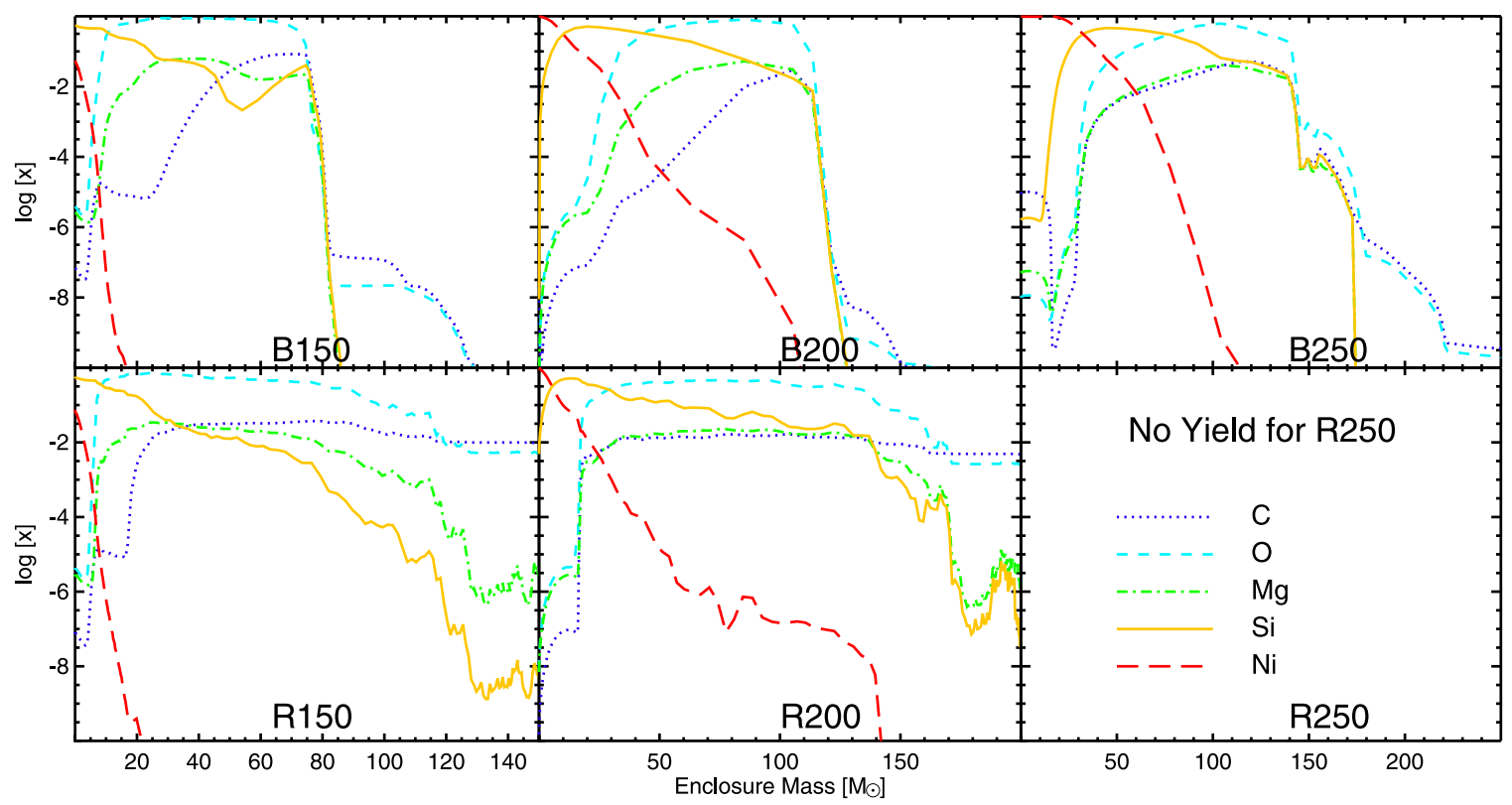

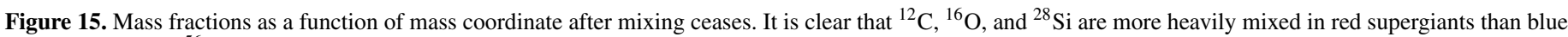
supergiants. Some ${ }^{56} \mathrm{Ni}$ is dredged up in the R200 run but it barely reaches the outer envelope.

(A color version of this figure is available in the online journal.) 
mixing of hot ash in the core. Second, burning time scales are so short compared to dynamical times that instabilities do not have time to become large enough to dredge material up from the core and drive mixing.

Later, when the SN shock reaches the helium layer, it burns ${ }^{4} \mathrm{He}$ into ${ }^{12} \mathrm{C}$, ${ }^{16} \mathrm{O}$, and ${ }^{56} \mathrm{Ni}$. The energy released creates pressure gradients opposite to the gradients in density and mass fractions, causing the formation of RT instabilities that also, in principle, could accelerate helium burning. But these instabilities do not survive for long because temperatures behind the shock rapidly fall as the star expands. About $100 \mathrm{~s}$ after core bounce, the postshock gas has cooled to $2 \times 10^{8} \mathrm{~K}$, and nuclear burning becomes too weak to drive further instability growth. They then become frozen in mass coordinate until the reverse shock forms. Although they may briefly enhance helium burning, it does not affect the speed of the shock or its energy.

Instabilities are too weak to dredge ${ }^{56} \mathrm{Ni}$ up from the core at early times in PSNe in either red or blue supergiants. Later, when instabilities with much larger amplitudes form in red supergiants because of the reverse shock, they still do not transport much ${ }^{56} \mathrm{Ni}$ up from lower depths and most of the energy from its decay is deposited locally in the ejecta. Our simulations therefore suggest that internal mixing in these events will probably not be visible in their observational signatures, in either the order that lines from metals appear in the spectra over time or the appearance of gamma rays from ${ }^{56} \mathrm{Ni}$ decay. The conclusions about the detectability of PSNe at high $z$ derived from prior 1D radiation hydrodynamics calculations therefore still hold. We note that if these explosions occur at high redshift, neither $\mathrm{X}$-rays and hard UV from shock breakout nor the leakage of gamma rays from ${ }^{56} \mathrm{Ni}$ at later times would be detected because of absorption by the neutral intergalactic medium and the outer layers of the Galaxy (Whalen et al. 2013a, 2013e).

We find that instability growth in Pop III PSNe is generally much weaker than in 15-40 $M_{\odot}$ Type II SNe, which exhibit rampant mixing (Chevalier 1976; Fryxell et al. 1991; Herant \& Woosley 1994; Joggerst et al. 2010). PSN shocks form at the edge of the carbon/oxygen core, which contains $40 \%$ of the mass of the star, and the envelope beyond it is not dense enough to foster as strong a reverse shock as in less massive stars. Unlike most PSNe, core-collapse SNe also exhibit fallback onto a central compact remnant that can enhance mixing. Stellar rotation can reduce the progenitor masses of PSNe (Chatzopoulos \& Wheeler 2012a; Chatzopoulos et al. 2013) but also tends to create blue supergiants in which there is less mixing. This point is important because recent cosmological simulations suggest that many Pop III stars may have been born with rotation speeds close to the breakup value (Stacy et al. 2011; Greif et al. 2012; Stacy et al. 2013).

The authors thank the anonymous referee for reviewing this manuscript and providing insightful comments and the members of the CCSE at LBNL for help with CASTRO. We are grateful to Volker Bromm, Dan Kasen, Lars Bildsten, John Bell, and Adam Burrows for useful discussions. K.C. was supported by the IAU-Gruber Fellowship, Stanwood Johnston Fellowship, and KITP Graduate Fellowship. A.H. was supported by a Future Fellowship from the Australian Research Council (ARC FT 120100363). S.W. acknowledges support by the DOE HEP Program under contract DESC0010676; the National Science Foundation (AST 0909129) and the NASA Theory Program (NNX14AH34G). D.J.W. acknowledges support by the Baden-Württemberg-Stiftung by contract research via the programme Internationale Spitzenforschung II (grant P-LS-SPII/18). All numerical simulations were done with allocations from the University of Minnesota Supercomputing Institute and the National Energy Research Scientific Computing Center. This work was supported by DOE grants DE-SC0010676, DE-AC02-05CH11231, DEGF02-87ER40328, and DE-FC02-09ER41618, and by NSF grants AST-1109394 and PHY02-16783. Work at LANL was done under the auspices of the National Nuclear Security Administration of the U.S. Department of Energy at Los Alamos National Laboratory under Contract No. DE-AC5206NA25396.

\section{REFERENCES}

Abel, T., Bryan, G. L., \& Norman, M. L. 2000, ApJ, 540, 39 Abel, T., Bryan, G. L., \& Norman, M. L. 2002, Sci, 295, 93 Abel, T., Wise, J. H., \& Bryan, G. L. 2007, ApJL, 659, L87 Agarwal, B., Khochfar, S., Johnson, J. L., et al. 2012, MNRAS, 425, 2854 Almgren, A. S., Beckner, V. E., Bell, J. B., et al. 2010, ApJ, 715, 1221 Alvarez, M. A., Wise, J. H., \& Abel, T. 2009, ApJL, 701, L133 Arnett, D., Meakin, C., \& Young, P. A. 2009, ApJ, 690, 1715 Baraffe, I., Heger, A., \& Woosley, S. E. 2001, ApJ, 550, 890 Barkat, Z., Rakavy, G., \& Sack, N. 1967, PhRvL, 18, 379 Bromm, V., Coppi, P. S., \& Larson, R. B. 1999, ApJL, 527, L5 Bromm, V., Coppi, P. S., \& Larson, R. B. 2002, ApJ, 564, 23 Chandrasekhar, S. (ed.) 1961, Hydrodynamic and Hydromagnetic Stability (Oxford: Oxford Univ. Press)

Chatzopoulos, E., \& Wheeler, J. C. 2012a, ApJ, 748, 42

Chatzopoulos, E., \& Wheeler, J. C. 2012b, ApJ, 760, 154

Chatzopoulos, E., Wheeler, J. C., \& Couch, S. M. 2013, ApJ, 776, 129

Chen, K., Heger, A., \& Almgren, A. S. 2011, CoPhC, 182, 254

Chen, K.-J., Heger, A., \& Almgren, A. 2012, JPhCS, 402, 012024

Chen, K.-J., Heger, A., \& Almgren, A. S. 2013, A\&C, 34, 70

Chen, K.-J., Woosley, S., Heger, A., Almgren, A., \& Whalen, D. 2014, ApJ, in press (arXiv:1402.4134)

Chevalier, R. A. 1976, ApJ, 207, 872

Cooke, J., Sullivan, M., Gal-Yam, A., et al. 2012, Natur, 491, 228

Cooke, R., \& Madau, P. 2014, arXiv:1405.7369

Crowther, P. A., Schnurr, O., Hirschi, R., et al. 2010, MNRAS, 408, 731

Davidson, K., \& Humphreys, R. M. 1997, ARA\&A, 35, 1

de Souza, R. S., Ishida, E. E. O., Johnson, J. L., Whalen, D. J., \& Mesinger, A. 2013, MNRAS, 436, 1555

de Souza, R. S., Ishida, E. E. O., Whalen, D. J., Johnson, J., \& Ferrara, A. 2014, MNRAS, 442, 1640

Dessart, L., Hillier, D. J., Waldman, R., Livne, E., \& Blondin, S. 2012, MNRAS, 426, L76

Dessart, L., Waldman, R., Livne, E., Hillier, D. J., \& Blondin, S. 2013, MNRAS, 428, 3227

Ekström, S., Meynet, G., Chiappini, C., Hirschi, R., \& Maeder, A. 2008, A\&A, 489,685

Fryxell, B., Arnett, D., \& Müller, E. 1991, ApJ, 367, 619

Gal-Yam, A., Mazzali, P., Ofek, E. O., et al. 2009, Natur, 462, 624

Glatzel, W., Fricke, K. J., \& El Eid, M. F. 1985, A\&A, 149, 413

Greif, T. H., Bromm, V., Clark, P. C., et al. 2012, MNRAS, 424, 399

Greif, T. H., Johnson, J. L., Klessen, R. S., \& Bromm, V. 2008, MNRAS, 387,1021

Greif, T. H., Springel, V., White, S. D. M., et al. 2011, ApJ, 737, 75

Heger, A., Woosley, S., Baraffe, I., \& Abel, T. 2002, in Lighthouses of the Universe: The Most Luminous Celestial Objects and Their Use for Cosmology, ed. M. Gilfanov, R. Sunyeav, \& E. Churazov (Berlin: Springer), 369

Heger, A., \& Woosley, S. E. 2002, ApJ, 567, 532

Heger, A., \& Woosley, S. E. 2010, ApJ, 724, 341

Heger, A., Woosley, S. E., Martínez-Pinedo, G., \& Langanke, K. 2001, ApJ, 560,307

Herant, M., \& Woosley, S. E. 1994, ApJ, 425, 814

Hirano, S., Hosokawa, T., Yoshida, N., et al. 2014, ApJ, 781, 60

Hosokawa, T., Omukai, K., Yoshida, N., \& Yorke, H. W. 2011, Sci, 334, 1250

Hummel, J. A., Pawlik, A. H., Milosavljević, M., \& Bromm, V. 2012, ApJ, 755,72

Humphreys, R. M., \& Davidson, K. 1979, ApJ, 232, 409

Jeon, M., Pawlik, A. H., Bromm, V., \& Milosavljević, M. 2014, MNRAS, 440,3778 
Jeon, M., Pawlik, A. H., Greif, T. H., et al. 2012, ApJ, 754, 34 Joggerst, C. C., Almgren, A., Bell, J., et al. 2010, ApJ, 709, 11 Joggerst, C. C., \& Whalen, D. J. 2011, ApJ, 728, 129

Johnson, J. L., Greif, T. H., \& Bromm, V. 2008, MNRAS, 388, 26

Johnson, J. L., Whalen, D. J., Even, W., et al. 2013a, ApJ, 775, 107

Johnson, J. L., Whalen, D. J., Fryer, C. L., \& Li, H. 2012, ApJ, 750, 66

Johnson, J. L., Whalen, D. J., Li, H., \& Holz, D. E. 2013b, ApJ, 771, 116

Karlsson, T., Bromm, V., \& Bland-Hawthorn, J. 2013, RvMP, 85, 809

Kasen, D., \& Bildsten, L. 2010, ApJ, 717, 245

Kasen, D., Woosley, S. E., \& Heger, A. 2011, ApJ, 734, 102

Kitayama, T., Yoshida, N., Susa, H., \& Umemura, M. 2004, ApJ, 613, 631

Krtička, J., \& Kubát, J. 2006, A\&A, 446, 1039

Kudritzki, R. 2000, in The First Stars, ed. A. Weiss, T. G. Abel, \& V. Hill (Berlin: Springer), 127

Latif, M. A., Schleicher, D. R. G., Schmidt, W., \& Niemeyer, J. 2013a, MNRAS, 433, 1607

Latif, M. A., Schleicher, D. R. G., Schmidt, W., \& Niemeyer, J. 2013b, MNRAS, 430, 588

Mackey, J., Bromm, V., \& Hernquist, L. 2003, ApJ, 586, 1

Marigo, P., Girardi, L., Chiosi, C., \& Wood, P. R. 2001, A\&A, 371, 152

Meakin, C. A., \& Arnett, D. 2007, ApJ, 667, 448

Meiksin, A., \& Whalen, D. J. 2013, MNRAS, 430, 2854

Mesler, R. A., Whalen, D. J., Smidt, J., et al. 2014, ApJ, 787, 91

Milosavljević, M., Bromm, V., Couch, S. M., \& Oh, S. P. 2009, ApJ, 698, 766

Moriya, T. J., Blinnikov, S. I., Tominaga, N., et al. 2013, MNRAS, 428, 1020

Nakamura, F., \& Umemura, M. 2001, ApJ, 548, 19

Ober, W. W., El Eid, M. F., \& Fricke, K. J. 1983, A\&A, 119, 61

O'Shea, B. W., \& Norman, M. L. 2007, ApJ, 654, 66

Pan, T., Kasen, D., \& Loeb, A. 2012, MNRAS, 422, 2701

Park, K., \& Ricotti, M. 2011, ApJ, 739, 2

Pawlik, A. H., Milosavljević, M., \& Bromm, V. 2011, ApJ, 731, 54

Pawlik, A. H., Milosavljević, M., \& Bromm, V. 2013, ApJ, 767, 59

Ritter, J. S., Safranek-Shrader, C., Gnat, O., Milosavljević, M., \& Bromm, V. 2012, ApJ, 761, 56

Safranek-Shrader, C., Milosavljevic, M., \& Bromm, V. 2014, MNRAS, 438, 1669

Santoro, F., \& Shull, J. M. 2006, ApJ, 643, 26

Scannapieco, E., Madau, P., Woosley, S., Heger, A., \& Ferrara, A. 2005, ApJ, 633, 1031

Schneider, R., Omukai, K., Inoue, A. K., \& Ferrara, A. 2006, MNRAS, 369,1437
Sedov, L. I. (ed.) 1959, Similarity and Dimensional Methods in Mechanics (New York: Academic)

Smith, B. D., \& Sigurdsson, S. 2007, ApJL, 661, L5

Smith, B. D., Turk, M. J., Sigurdsson, S., O’Shea, B. W., \& Norman, M. L. 2009, ApJ, 691, 441

Stacy, A., Bromm, V., \& Loeb, A. 2011, MNRAS, 413, 543

Stacy, A., Greif, T. H., \& Bromm, V. 2010, MNRAS, 403, 45

Stacy, A., Greif, T. H., Klessen, R. S., Bromm, V., \& Loeb, A. 2013, MNRAS, 431, 1470

Stacy, A., Pawlik, A. H., Bromm, V., \& Loeb, A. 2012, MNRAS, 421, 894

Steiner, O., \& Gautschy, A. (ed.) 1998, Computational Methods for Astrophysical Fluid Flow (Berlin: Springer)

Stringfellow, G. S., \& Woosley, S. E. 1988, in Origin and Distribution of the Elements, ed. G. J. Mathews (Singapore: World Scientific Publishing), 467

Timmes, F. X. 1999, ApJS, 124, 241

Timmes, F. X., \& Swesty, F. D. 2000, ApJS, 126, 501

Turk, M. J., Abel, T., \& O’Shea, B. 2009, Sci, 325, 601

Vink, J. S., de Koter, A., \& Lamers, H. J. G. L. M. 2001, A\&A, 369, 574

Weaver, T. A., Zimmerman, G. B., \& Woosley, S. E. 1978, ApJ, 225, 1021

Whalen, D., Abel, T., \& Norman, M. L. 2004, ApJ, 610, 14

Whalen, D. J., Even, W., Frey, L. H., et al. 2013a, ApJ, 777, 110

Whalen, D. J., Even, W., Lovekin, C. C., et al. 2013b, ApJ, 768, 195

Whalen, D. J., Even, W., Smidt, J., et al. 2013c, arXiv:1312.5360

Whalen, D. J., Even, W., Smidt, J., et al. 2013d, ApJ, 778, 17

Whalen, D. J., \& Fryer, C. L. 2012, ApJL, 756, L19

Whalen, D. J., Fryer, C. L., Holz, D. E., et al. 2013e, ApJL, 762, L6

Whalen, D. J., Joggerst, C. C., Fryer, C. L., et al. 2013f, ApJ, 768, 95

Whalen, D. J., Johnson, J. L., Smidt, J., et al. 2013g, ApJ, 777, 99

Whalen, D. J., Johnson, J. L., Smidt, J., et al. 2013h, ApJ, 774, 64

Whalen, D. J., Smidt, J., Even, W., et al. 2014, ApJ, 781, 106

Whalen, D. J., Smidt, J., Johnson, J. L., et al. 2013i, arXiv:1312.6330

Wise, J. H., Turk, M. J., Norman, M. L., \& Abel, T. 2012, ApJ, 745, 50

Woodward, P. R., Herwig, F., \& Lin, P.-H. 2013, arXiv:1307.3821

Woosley, S. E. 2010, ApJL, 719, L204

Woosley, S. E., Heger, A., Roberts, L., \& Hoffman, R. D. 2010, in IAU Symp. 265, Chemical Abundances in the Universe: Connecting First Stars to Planets, ed. K. Cunha, M. Spite, \& B. Barbuy (Cambridge: Cambridge Univ. Press),

Yoshida, N., Omukai, K., \& Hernquist, L. 2008, Sci, 321, 669

Zhang, W., Howell, L., Almgren, A., Burrows, A., \& Bell, J. 2011, ApJS, 196, 20 